\title{
KERNEL-FUNCTION BASED ALGORITHMS FOR SEMIDEFINITE OPTIMIZATION*
}

\author{
M. EL GHAMi ${ }^{1}$, Y.Q. BAI ${ }^{2}$ AND C. ROOS $^{3}$
}

\begin{abstract}
Recently, Y.Q. Bai, M. El Ghami and C. Roos [3] introduced a new class of so-called eligible kernel functions which are defined by some simple conditions. The authors designed primal-dual interiorpoint methods for linear optimization (LO) based on eligible kernel functions and simplified the analysis of these methods considerably. In this paper we consider the semidefinite optimization (SDO) problem and we generalize the aforementioned results for LO to SDO. The iteration bounds obtained are analogous to the results in [3] for LO.
\end{abstract}

Keywords. Semidefinite optimization, interior-point methods, primaldual method, complexity.

Mathematics Subject Classification. 90C22, 90C31.

\section{INTRODUCTION}

In this paper we deal with the semidefinite optimization (SDO) problem in the standard form:

$$
\text { (P) } \quad p^{*}=\inf _{X \in \mathbf{S}^{n}}\left\{\operatorname{Tr}(C X): \operatorname{Tr}\left(A_{i} X\right)=b_{i}(1 \leq i \leq m), X \succeq 0\right\}
$$

Received October 10, 2007. Accepted January 27, 2009.

* The second author kindly acknowledges support of the grants of National Science Foundation of China (No. 10771133) and the Key Disciplines of Shanghai Municipality (S30104).

1 Department of Informatics, University of Bergen,Post Box 78035020 Bergen, Norway; melghami@ii.uib.no

2 Department of Mathematics, Shanghai University, Shanghai, 200444, P.R. China; yqbai@shu. edu.cn

${ }^{3}$ Faculty of Electrical Engineering, Mathematics, and Computer Science, Delft University of

Technology, P.O. Box 5031, 2600 GA Delft, The Netherlands; C.Roos@ewi.tudelft.nl 
and its dual problem:

$$
\text { (D) } d^{*}=\sup _{y, S}\left\{b^{T} y: \sum_{i=1}^{m} y_{i} A_{i}+S=C, S \succeq 0\right\} \text {, }
$$

where $C \in \mathbf{S}^{n}$ and $A_{i} \in \mathbf{S}^{n}, i=1, \ldots, m, b \in \mathbf{R}^{m} . \quad X \succeq 0$ means that $X$ is a symmetric positive semidefinite matrix. Without loss of generality we assume that the matrices $A_{i}$ are linearly independent. Interior-point methods (IPMs) provide a powerful approach for solving SDO problems. A comprehensive list of publications on this topic can be found in the SDO homepage maintained by Alizadeh [1]. The pioneering works in this direction are due to Alizadeh [1,2] and Nesterov and Nemirovskii [8]. Most IPMs for SDO can be viewed as natural extensions of IPMs for linear optimization (LO) and have similar polynomial complexity results. However, obtaining a valid search direction in SDO case is much more difficult than in the $\mathrm{LO}$ case.

In this paper we present the approach of using kernel function to determine the search directions and to design primal-dual IPMs for solving SDO problems.

Our aim in this paper is to show that the approach presented in [3] for LO, which is based on so-called eligible kernel functions, can be extended in a natural way to SDO, thus yielding a wide class of new methods for SDO. For another class, of the so-called self-regular kernel functions, this has already been done [9]. Just as in the LO case, the new methods have the same iteration complexity for small-update methods, but the iteration complexity is better for large-update methods.

This paper is organized as follows. In Section 2 we recall the notion of a kernel function, and the barrier function for SDO induced by a kernel function, and we show how any kernel function defines in a natural way a primal-dual interior-point algorithm. In Section 3, following [3] we introduce the class of eligible kernel functions. We also recall some results from [3] that are useful in the current context. Section 3.2 is devoted to properties of an eligible kernel function and its barrier function that we need in the analysis of the algorithm. We find a default value for the step size, an upper bound for the decrease of the barrier function during one iteration, etc. The main result is that the complexity of the algorithm can be obtained by the same simple scheme that was presented in [3] for LO; the only input for the scheme, which is given in Section 3.3, is the kernel function and the dimension $n$ of the problem that we want to solve. Finally, some concluding remarks follow in Section 4.

We use the following notational conventions. Throughout, $\|\cdot\|$ denotes the 2-norm of a vector. The nonnegative and the positive orthants are denoted as $\mathbf{R}_{+}^{n}$ and $\mathbf{R}_{++}^{n}$, respectively, and $\mathbf{S}^{n}, \mathbf{S}_{+}^{n}$, and $\mathbf{S}_{++}^{n}$ denote the cone of symmetric, positive semidefinite and positive definite $n \times n$ matrices, respectively. For any $V \in \mathbf{S}^{n}$, we denote by $\lambda(V)$ the vector of eigenvalues of $V$ arranged in nondecreasing order, that is, $\lambda_{1}(V) \leq \lambda_{2}(V) \leq, \ldots, \lambda_{n}(V)$.

Finally, if $z \in \mathbf{R}_{+}^{n}$ and $f: \mathbf{R}_{+} \rightarrow \mathbf{R}_{+}$, then $f(z)$ denotes the vector in $\mathbf{R}_{+}^{n}$ whose $i$-th component is $f\left(z_{i}\right)$, with $1 \leq i \leq n$. 


\section{Algorithms BASED ON KeRnel FUnCtions}

\subsection{Preliminaries}

We assume that $(P)$ and $(D)$ satisfy the interior-point condition (IPC), i.e., there exists $X^{0} \succ 0$ and $\left(y^{0}, S^{0}\right)$ with $S^{0} \succ 0$ such that $X^{0}$ is feasible for $(P)$ and $\left(y^{0}, S^{0}\right)$ is feasible for $(D)$. Moreover, we may assume that $X^{0}=S^{0}=E$, where $E$ is the identity matrix. In that case, for any $\mu>0$ there exist a unique primal feasible $X$ and unique dual feasible $y$ and $S$ such that

$$
X S=\mu E .
$$

This solution is denoted by $(X(\mu), y(\mu), S(\mu)) ; X(\mu)$ is called the $\mu$-center of $(P)$ and $(y(\mu), S(\mu))$ is the $\mu$-center of $(D)$. The set of $\mu$-centers (with $\mu>0$ ) defines a homotopy path, which is called the central path of $(P)$ and $(D)[4,9]$.

As is well known, the definition of search directions for SDO requires some symmetrization scheme. In this paper we use the scheme due to Nesterov and Todd, which uses the positive definite matrix

$$
P:=X^{\frac{1}{2}}\left(X^{\frac{1}{2}} S X^{\frac{1}{2}}\right)^{-\frac{1}{2}} X^{\frac{1}{2}}=S^{-\frac{1}{2}}\left(S^{\frac{1}{2}} X S^{\frac{1}{2}}\right)^{\frac{1}{2}} S^{-\frac{1}{2}}
$$

the last equality can be verified by elementary means. Let $X \succ 0$ be primal and $S \succ 0$ dual feasible, and let $D=P^{\frac{1}{2}}$, where $P^{\frac{1}{2}}$ denotes the symmetric square root of $P$. Then the matrix $D$ can be used to scale $X$ and $S$ to the same matrix $V$, which is defined by $[4,11]$ :

$$
V:=\frac{1}{\sqrt{\mu}} D^{-1} X D^{-1}=\frac{1}{\sqrt{\mu}} D S D=\frac{1}{\sqrt{\mu}}\left(D^{-1} X S D\right)^{\frac{1}{2}} .
$$

Obviously the matrices $D$ and $V$ are symmetric and positive definite.

\subsection{KERNEL FUNCTIONS AND THEIR BARRIER FUNCTIONS}

We call the univariate function $\psi:(0, \infty) \rightarrow[0, \infty)$ a kernel function if $\psi$ is three times differentiable and the following conditions are satisfied.

(i) $\psi^{\prime}(1)=\psi(1)=0$;

(ii) $\psi^{\prime \prime}(t)>0$, for all $t>0$;

(iii) $\lim _{t \downarrow 0} \psi(t)=\lim _{t \rightarrow \infty} \psi(t)=\infty$.

Clearly, $(i)$ and $(i i)$ say that $\psi(t)$ is a nonnegative strictly convex function which is minimal at $t=1$, with $\psi(1)=0$. Note that $\psi(t)$ is completely determined by its second derivative, because we have

$$
\psi(t)=\int_{1}^{t} \int_{1}^{\xi} \psi^{\prime \prime}(\zeta) \mathrm{d} \zeta \mathrm{d} \xi
$$


Because of property (iii) we say that $\psi(t)$ has the barrier property.

Given a kernel function $\psi$, we recall the definition of a matrix function $[7,10]$.

Definition 2.1. Let $X$ be a positive definite (and hence symmetric) matrix, and let

$$
X=Q_{X}^{-1} \operatorname{diag}\left(\lambda_{1}(X), \lambda_{2}(X), \ldots, \lambda_{n}(X)\right) Q_{X}
$$

be an eigenvalue decomposition of $X$, where $\lambda_{i}(X), 1 \leq i \leq n$, denote the eigenvalues of $X$, and $Q_{X}$ is orthogonal. Following [9], the matrix function $\psi(X): \mathbf{S}^{n} \rightarrow \mathbf{S}^{n}$ is defined by

$$
\psi(X)=Q_{X}^{-1} \operatorname{diag}\left(\psi\left(\lambda_{1}(X)\right), \psi\left(\lambda_{2}(X)\right), \ldots, \psi\left(\lambda_{n}(X)\right)\right) Q_{X}
$$

and the barrier function $\Psi(X): \mathbf{S}^{n} \rightarrow \mathbf{R}$ induced by $\psi$ is defined by

$$
\Psi(X):=\sum_{i=1}^{n} \psi\left(\lambda_{i}(X)\right)=\operatorname{Tr}(\psi(X)) .
$$

In this paper, when we use the function $\psi($.$) and its first three derivatives \psi^{\prime}($.$) ,$ $\psi^{\prime \prime}($.$) , and \psi^{\prime \prime \prime}($.$) without any specification, it denotes a matrix function if the$ argument is a matrix and a univariate function (from $\mathbf{R}$ to $\mathbf{R}$ ) if the argument is in $\mathbf{R}$.

\subsection{KERNEL-FUnCtion BASED SEARCH DIRECTIONS}

Following [9] we now describe the kernel-function-based approach to SDO. Given the kernel function $\psi(t)$ and the associated $\psi(V)$ and $\psi^{\prime}(V)$ as defined in Definition 2.1, just as in [9] we define the scaled search direction by the following linear system:

$$
\begin{aligned}
\operatorname{Tr}\left(\bar{A}_{i} D_{X}\right) & =0, \quad i=1 \ldots, m, \\
\sum_{i=1}^{m} \Delta y_{i} \bar{A}_{i}+D_{S} & =0, \\
D_{X}+D_{S} & =-\psi^{\prime}(V),
\end{aligned}
$$

where

$$
\bar{A}_{i}:=D A_{i} D, \quad i=1,2, \ldots, m,
$$

Having $D_{X}$ and $D_{S}$, we find displacements $\Delta X$ and $\Delta S$ in the $X$ and $S$-spaces from

$$
\Delta X:=\mu D D_{X} D ; \quad \Delta S:=\mu D^{-1} D_{S} D^{-1} .
$$

One may easily understand that $D_{X}$ and $D_{S}$, and also $\Delta X$ and $\Delta S$ are orthogonal. We therefore have

$$
\operatorname{Tr}\left(D_{X} D_{S}\right)=0 .
$$




\section{Generic Primal-Dual Algorithm for SDO}

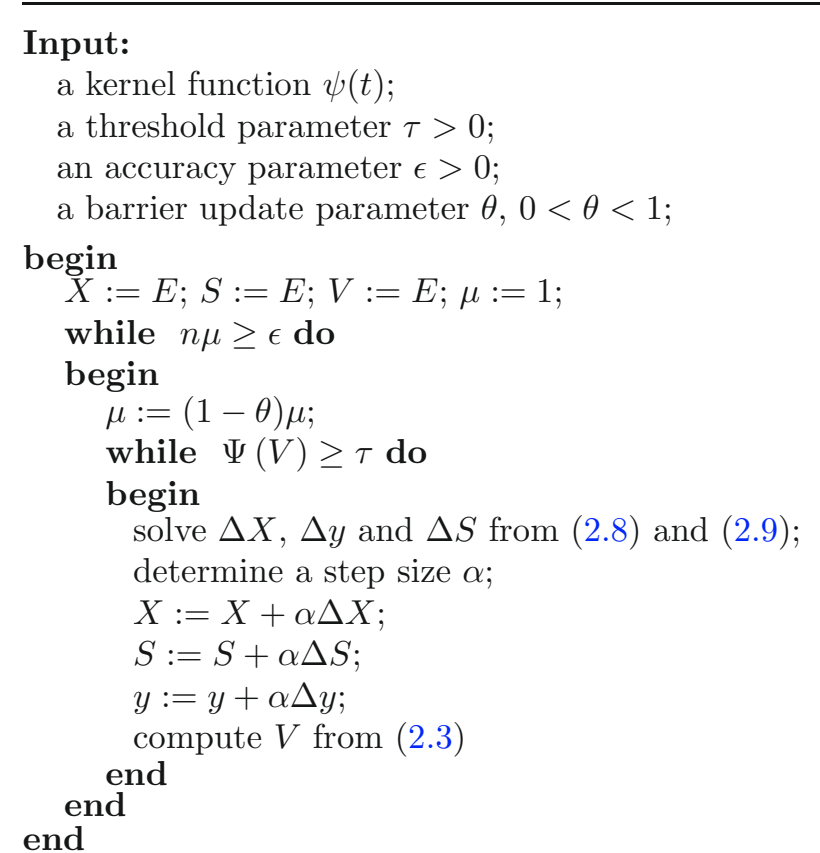

FiguRE 1. Generic primal-dual interior-point algorithm for SDO.

\subsection{GENERIC PRIMAL-DUAL INTERIOR-POINT ALGORITHM}

The algorithm considered in this paper is described in Figure 1. Note that we initialize the algorithm with $X=S=E$. Since then $X S=1 \cdot E$, this means that $X=X(1)$ and $S=S(1)$, and hence $V=E$, and therefore $\Psi(V)=0$.

Given the kernel function $\psi(t)$, just as in the case of linear optimization, the parameters $\tau, \theta$, and the step size $\alpha$ should be chosen in such a way that the algorithm is 'optimized' in the sense that the number of iterations required by the algorithm is as small as possible. Obviously, the resulting iteration bound will depend on the kernel function, and our main task is to find a kernel function that minimizes the iteration bound.

In the analysis of the algorithm we also use a norm-based proximity measure $\delta(V)$, which is defined by

$$
\delta(V)=\frac{1}{2}\left\|\psi^{\prime}(V)\right\|=\frac{1}{2} \sqrt{\sum_{i=1}^{n} \psi^{\prime}\left(\lambda_{i}(V)\right)^{2}}=\frac{1}{2}\left\|D_{X}+D_{S}\right\| .
$$




\section{Eligible KeRnel FUnCtions}

\subsection{Definition}

In this paper we only deal with so-called eligible kernel functions. Such kernel functions were introduced in [3] and are characterized by the fact that the following four conditions are satisfied

$$
\begin{aligned}
t \psi^{\prime \prime}(t)+\psi^{\prime}(t) & >0, & t<1, & \\
\psi^{\prime \prime \prime}(t) & <0, & t>0, & \\
2 \psi^{\prime \prime}(t)^{2}-\psi^{\prime}(t) \psi^{\prime \prime \prime}(t) & >0, & t<1, & \\
\psi^{\prime \prime}(t) \psi^{\prime}(\beta t)-\beta \psi^{\prime}(t) \psi^{\prime \prime}(\beta t) & >0, & t>1, & \beta>1 .
\end{aligned}
$$

By way of example we presented in [3], Table 6.4, seven eligible kernel functions, and the iteration bound for the corresponding algorithm for LO for each of these functions. As we show in this paper, exactly the same bounds can obtained for SDO.

In the sequel we denote by $\varrho:[0, \infty) \rightarrow[1, \infty)$ and $\rho:[0, \infty) \rightarrow(0,1]$ the inverse functions of $\psi(t)$ for $t \geq 1$, and $-\frac{1}{2} \psi^{\prime}(t)$ for $t \leq 1$, respectively. In other words

$$
\begin{aligned}
s=\psi(t) & \Leftrightarrow \quad t=\varrho(s), \quad t \geq 1, \\
s=-\frac{1}{2} \psi^{\prime}(t) & \Leftrightarrow \quad t=\rho(s), \quad t \leq 1 .
\end{aligned}
$$

Let us also mention here that at some places we apply the function $\Psi$ to a positive vector $v$. The definition is compatible with Definition 2.1 when identifying the vector $v$ with its diagonal matrix $\operatorname{diag}(v)$ and applying $\Psi$ to this matrix to obtain

$$
\Psi(v)=\sum_{i=1}^{n} \psi\left(v_{i}\right), \quad v \in \mathbf{R}_{++}^{n} .
$$

\subsection{Properties of $\boldsymbol{\Psi}(\boldsymbol{V})$ And $\boldsymbol{\delta}(\boldsymbol{V})$}

In this subsection we extend Theorem 3.2 and Theorem 4.9 in [3] to positive definite matrices. Let us recall from [3] that these theorems follows from conditions (3.1-d) and (3.1-b), respectively.

Theorem 3.1. With @ as defined in (3.2), we have for any positive definite matrix $V$, and any $\beta>1$,

$$
\Psi(\beta V) \leq n \psi\left(\beta \varrho\left(\frac{\Psi(V)}{n}\right)\right) .
$$

Proof. Let $v_{i}:=\lambda_{i}(V), 1 \leq i \leq n$. Then $v>0$ and

$$
\Psi(\beta V)=\sum_{i=1}^{n} \psi\left(\beta \lambda_{i}(V)\right)=\sum_{i=1}^{n} \psi\left(\beta v_{i}\right)=\Psi(\beta v) .
$$


Using [3], Theorem 3.2, we may write

$$
\Psi(\beta v) \leq n \psi\left(\beta \varrho\left(\frac{\Psi(v)}{n}\right)\right)=n \psi\left(\beta \varrho\left(\frac{\sum_{i=1}^{n} \psi\left(\lambda_{i}(V)\right)}{n}\right)\right)=n \psi\left(\beta \varrho\left(\frac{\Psi(V)}{n}\right)\right) .
$$

This proves the theorem.

The next theorem gives a lower bound on the norm-based proximity measure $\delta(V)$, as defined by (2.11), in terms of $\Psi(V)$. Observe that since $\Psi(V)$ is strictly convex and attains its minimal value zero at $V=E$, we have

$$
\Psi(V)=0 \quad \Leftrightarrow \quad \delta(V)=0 \quad \Leftrightarrow \quad V=E .
$$

Theorem 3.2. Let $\varrho$ be as defined in (3.2). Then $\delta(V) \geq \frac{1}{2} \psi^{\prime}(\varrho(\Psi(V)))$.

Proof. The statement in the lemma is obvious if $V=E$ since then $\delta(V)=\Psi(V)=$ 0 . Otherwise we have $\delta(V)>0$ and $\Psi(V)>0$. To deal with the nontrivial case we again let $v_{i}:=\lambda_{i}(V), 1 \leq i \leq n$. Then $v>0$ and

$$
\frac{1}{4} \sum_{i=1}^{n} \psi^{\prime}\left(\lambda_{i}(V)\right)^{2}=\frac{1}{4} \sum_{i=1}^{n} \psi^{\prime}\left(v_{i}\right)^{2}=\delta(v)^{2}
$$

Using [3], Theorem 4.9, we get

$$
\delta(v) \geq \frac{1}{2} \psi^{\prime}(\varrho(\Psi(v)))=\frac{1}{2} \psi^{\prime}\left(\varrho\left(\sum_{i=1}^{n} \psi\left(\lambda_{i}(V)\right)\right)\right)=\frac{1}{2} \psi^{\prime}(\varrho(\Psi(V))) .
$$

This completes the proof of the theorem.

\subsection{AnAlysis OF the ALGORIthM}

In the analysis of the algorithm the concept of exponential convexity $[3,5,6]$ is again a crucial ingredient. In this section we derive a default value for the step size and we obtain an upper bound for the decrease in $\Psi(V)$ during a Newton step. A consequence of condition (3.1-a) is that any eligible kernel function is exponentially convex [9], equation (2.10):

$$
\psi\left(\sqrt{t_{1} t_{2}}\right) \leq \frac{1}{2}\left(\psi\left(t_{1}\right)+\psi\left(t_{2}\right)\right), \quad \forall t_{1}>0, \forall t_{2}>0
$$

As was shown in [9], Proposition 5.2.6, this implies the following inequality, which is crucial for our purpose.

$$
\Psi\left(\left(V_{1}^{\frac{1}{2}} V_{2} V_{1}^{\frac{1}{2}}\right)^{\frac{1}{2}}\right) \leq \frac{1}{2}\left(\Psi\left(V_{1}\right)+\Psi\left(V_{2}\right)\right), \quad \forall V_{1} \succ 0, \forall V_{2} \succ 0 .
$$


After a damped step with step size $\alpha$, using (2.9) and (2.3) we have

$$
\begin{aligned}
& X_{+}=X+\alpha \Delta X=X+\alpha \sqrt{\mu} D D_{X} D=\sqrt{\mu} D\left(V+\alpha D_{X}\right) D \\
& S_{+}=S+\alpha \Delta S=X+\alpha \sqrt{\mu} D^{-1} D_{S} D^{1}=\sqrt{\mu} D^{-1}\left(V+\alpha D_{S}\right) D^{-1} .
\end{aligned}
$$

By (2.3), the $V$-matrix corresponding to $X_{+}$and $S_{+}$with respect to $\mu$, is given by $V_{+}=\frac{1}{\sqrt{\mu}}\left(D^{-1} X_{+} S_{+} D\right)^{\frac{1}{2}}$. From this we deduce that $V_{+}^{2}$ is unitarily similar to the matrix $X_{+}^{\frac{1}{2}} S_{+} X_{+}^{\frac{1}{2}}$ and hence the eigenvalues of $V_{+}$are the same as those of the matrix

$$
\tilde{V}_{+}:=\left[\left(V+\alpha D_{X}\right)^{\frac{1}{2}}\left(V+\alpha D_{S}\right)\left(V+\alpha D_{X}\right)^{\frac{1}{2}}\right]^{\frac{1}{2}} .
$$

By the definition of $\Psi$ we have $\Psi\left(V_{+}\right)=\Psi\left(\tilde{V}_{+}\right)$. Our aim is to find $\alpha$ such that the decrement

$$
f(\alpha):=\Psi\left(V_{+}\right)-\Psi(V)=\Psi\left(\tilde{V}_{+}\right)-\Psi(V),
$$

is as small as possible. Due to (3.4) it follows that

$$
\begin{aligned}
\Psi\left(\tilde{V}_{+}\right)=\Psi\left(\left[\left(V+\alpha D_{X}\right)^{\frac{1}{2}}\left(V+\alpha D_{S}\right)\left(V+\alpha D_{X}\right)^{\frac{1}{2}}\right]^{\frac{1}{2}}\right) & \\
& \leq \frac{1}{2}\left[\Psi\left(V+\alpha D_{X}\right)+\Psi\left(V+\alpha D_{S}\right)\right] .
\end{aligned}
$$

Therefore we have $f(\alpha) \leq f_{1}(\alpha)$, where

$$
f_{1}(\alpha):=\frac{1}{2}\left[\Psi\left(V+\alpha D_{X}\right)+\Psi\left(V+\alpha D_{S}\right)\right]-\Psi(V) .
$$

Note that is $f_{1}(\alpha)$ is convex in $\alpha$, since $\Psi$ is convex. Obviously, $f(0)=f_{1}(0)=0$.

Taking the derivative to $\alpha$, we get

$$
f_{1}^{\prime}(\alpha)=\frac{1}{2} \operatorname{Tr}\left(\psi^{\prime}\left(V+\alpha D_{X}\right) D_{X}+\psi^{\prime}\left(V+\alpha D_{S}\right) D_{S}\right) .
$$

This gives, using the last equality in (2.8), and also (2.11),

$$
f_{1}^{\prime}(0)=\frac{1}{2} \operatorname{Tr}\left(\psi^{\prime}(V)\left(D_{X}+D_{S}\right)\right)=-\frac{1}{2} \operatorname{Tr}\left(\psi^{\prime}(V)^{2}\right)=-2 \delta(V)^{2} .
$$

Differentiating once more, we obtain

$$
f_{1}^{\prime \prime}(\alpha)=\frac{1}{2} \operatorname{Tr}\left(\psi^{\prime \prime}\left(V+\alpha D_{X}\right) D_{X}^{2}+\psi^{\prime \prime}\left(V+\alpha D_{S}\right) D_{S}^{2}\right) .
$$

Below we use the shorthand notation

$$
\delta:=\delta(V) .
$$

Starting from (3.7) and using the same arguments as in [12] one obtains the following lemma, which is cited without proof. 
Lemma 3.3 (Lemma 5.19 in [12]). One has

$$
f_{1}^{\prime \prime}(\alpha) \leq 2 \delta^{2} \psi^{\prime \prime}\left(\lambda_{1}(V)-2 \alpha \delta\right) \text {. }
$$

Putting $v_{i}=\lambda_{i}(X), 1 \leq i \leq n$, we have

$$
f_{1}^{\prime \prime}(\alpha) \leq 2 \delta^{2} \psi^{\prime \prime}\left(v_{1}-2 \alpha \delta\right)
$$

which is the same inequality as in Lemma 4.1 in [3]. From this stage on we can apply word-by-word the same arguments as in [3] for the LO case to obtain the following results.

Lemma 3.4 (Lem. 4.2 in [3]). One has $f_{1}^{\prime}(\alpha) \leq 0$ if $\alpha$ satisfies the inequality

$$
-\psi^{\prime}\left(\lambda_{1}(V)-2 \alpha \delta\right)+\psi^{\prime}\left(\lambda_{1}(V)\right) \leq 2 \delta
$$

Lemma 3.5 (Lem. 4.3 in [3]). With $\rho$ as defined in (3.3), the largest possible solution of inequality (3.8) is the step size

$$
\bar{\alpha}:=\frac{\rho(\delta)-\rho(2 \delta)}{2 \delta}
$$

Lemma 3.6. Let $\rho$ and $\bar{\alpha}$ be as defined in Lemma 3.5. Then

$$
\frac{1}{\psi^{\prime \prime}(\rho(2 \delta))} \leq \bar{\alpha} \leq \frac{1}{\psi^{\prime \prime}(\rho(\delta))} .
$$

The left inequality in (3.10) is in [3], Lemma 4.4, the proof of the right inequality goes in a similar way. As in the LO case, we use

$$
\widetilde{\alpha}=\frac{1}{\psi^{\prime \prime}(\varrho(2 \delta))},
$$

as the default step size. By Lemma 3.6 we have $\widetilde{\alpha} \leq \bar{\alpha}$.

Theorem 3.7 (Thm. 4.6 in [3]). Let $\rho$ be as defined in (3.3) and $\widetilde{\alpha}$ as in (3.10). Then

$$
f(\widetilde{\alpha}) \leq-\frac{\delta^{2}}{\psi^{\prime \prime}(\rho(2 \delta))} .
$$

Just as in the LO case, the right-hand side expression in (3.11) is monotonically decreasing in $\delta$, due to (3.1-c). Hence, by using the results of Theorems 3.7 and 3.2 we obtain

$$
f(\tilde{\alpha}) \leq-\frac{\left(\psi^{\prime}(\varrho(\Psi(V)))^{2}\right.}{4 \psi^{\prime \prime}\left(\rho\left(\psi^{\prime}(\varrho(\Psi(V)))\right)\right.} .
$$

This upper bound for the decrease in $\Psi(V)$ during an inner iteration is completely in terms of $\psi(t)$, its first and second derivatives $\psi^{\prime}(t)$ and $\psi^{\prime \prime}(t)$, and the inverse functions $\rho$ and $\varrho$. 
Step 0: Specify a kernel function $\psi(t)$; an update parameter $\theta, 0<\theta<1$; a threshold parameter $\tau$; and an accuracy parameter $\epsilon$.

Step 1: Solve the equation $-\frac{1}{2} \psi^{\prime}(t)=s$ to get $\rho(s)$, the inverse function of $-\frac{1}{2} \psi^{\prime}(t), t \in(0,1]$. If the equation is hard to solve, derive a lower bound for $\rho(s)$.

Step 2: Calculate the decrease of $\Psi(v)$ during an inner iteration in terms of $\delta$ for the default step size $\tilde{\alpha}$ from

$$
f(\tilde{\alpha}) \leq-\frac{\delta^{2}}{\psi^{\prime \prime}(\rho(2 \delta))} .
$$

Step 3: Solve the equation $\psi(t)=s$ to get $\varrho(s)$, the inverse function of $\psi(t), t \geq 1$. If the equation is hard to solve, derive lower and upper bounds for $\varrho(s)$.

Step 4: Derive a lower bound for $\delta$ in terms of $\Psi(v)$ by using

$$
\delta(V) \geq \frac{1}{2} \psi^{\prime}(\varrho(\Psi(V))
$$

Step 5: Using the results of step 3 and step 4 find a valid inequality of the form

$$
f(\tilde{\alpha}) \leq-\kappa \Psi(V)^{1-\gamma}
$$

for some positive constants $\kappa$ and $\gamma$, with $\gamma \in(0,1]$ as small as possible.

Step 6: Calculate the upper bound of $\Psi_{0}$ from

$$
\Psi_{0} \leq L_{\psi}(n, \theta, \tau)=n \psi\left(\frac{\varrho\left(\frac{\tau}{n}\right)}{\sqrt{1-\theta}}\right) \leq \frac{n}{2} \psi^{\prime \prime}(1)\left(\frac{\varrho\left(\frac{\tau}{n}\right)}{\sqrt{1-\theta}}-1\right)^{2} .
$$

Step 7: Derive an upper bound for the total number of iterations by using that

$$
\frac{\Psi_{0}^{\gamma}}{\theta \kappa \gamma} \log \frac{n}{\epsilon}
$$

is an upper bound for this number.

Step 8: Set $\tau=O(n)$ and $\theta=\Theta(1)$ to calculate a complexity bound for largeupdate methods, and set $\tau=O(1)$ and $\theta=\Theta\left(\frac{1}{\sqrt{n}}\right)$ to calculate a complexity bound for small-update methods.

FIGURE 2. Scheme for analyzing a kernel-function-based algorithm.

As a result, the further analysis boils down to exactly the same analysis as given in [3] for the LO case. We do not wish to repeat this analysis here, but refer to [3]. As a result the computations required to get iteration bounds can be performed in a systematic way by using the scheme in Figure 2, which is essentially the same scheme as was presented in [3], Section 6.1, for the LO case.

\section{Concluding Remarks}

In this paper we extended the results obtained for kernel-function-based IPMs in [3] for LO to SDO. We have seen that at some point (after Lem. 3.3 in this paper) the analysis boils down to exactly the same analysis as for the LO case. 
As a consequence, the resulting iteration bounds for semidefinite optimization are the same as the bounds that were obtained in [3] for LO.

Apart from the topics for future research that were mentioned in [3], there is one interesting topic which is of special interest for semidefinite optimization: the search direction used in this paper is based on the Nesterov-Todd symmetrization scheme and it is natural to ask whether or not other existing symmetrization schemes can be used in the kernel-function-based framework.

\section{REFERENCES}

[1] F. Alizadeh, Combinatorial optimization with interior point methods and semi-definite matrices. Ph.D. thesis, University of Minnesota, Minneapolis, Minnesota, USA (1991).

[2] F. Alizadeh, Interior point methods in semidefinite programming with applications to combinatorial optimization. SIAM J. Optim. 5 (1995) 13-51.

[3] Y.Q. Bai, M. El Ghami and C. Roos, A comparative study of kernel functions for primal-dual interior-point algorithms in linear optimization. SIAM J. Optim. 15 (2004) 101-128.

[4] E. de Klerk, Aspects of semidefinite programming, Applied Optimization 65. Kluwer Academic Publishers, Dordrecht, The Netherlands (2002).

[5] M. El Ghami, New primal-dual interior-point methods based on kernel functions. Ph.D. thesis, TU Delft, The Netherlands (2005).

[6] M. El Ghami and C. Roos, Generic primal-dual interior point methods based on a new Kernel function. RAIRO-Oper. Res. 42 (2008) 199-213.

[7] R.A. Horn and C.R. Johnson, Matrix analysis. Cambridge University Press, Cambridge, UK (1985).

[8] Y.E. Nesterov and A.S. Nemirovskii, Interior point polynomial methods in convex programming: theory and algorithms. SIAM Publications, SIAM, Philadelphia, USA (1993).

[9] J. Peng, C. Roos and T. Terlaky, Self-regularity, a new paradigm for primal-dual interiorpoint algorithms. Princeton University Press (2002).

[10] W. Rudin, Principles of mathematical analysis. Mac-Graw Hill Book Company, New York (1978).

[11] J.F. Sturm and S. Zhang, Symmetric primal-dual path following algorithms for semidefinite programming. Appl. Num. Math. 29 (1999) 301-315.

[12] G.Q. Wang, Y.Q. Bai and C. Roos, Primal-dual interior-point algorithms for semidefinite optimization based on a simple kernel function. J. Math. Model. Algorithms 4 (2005) 409-433. 